\section{Oxytocin analogues have potential in relieving chronic abdominal pain}

New research demonstrates that stable oxytocin analogues could have potential as a treatment for chronic abdominal pain. Speaking about the study's main findings, author Paul Alewood explains: "It appears that the oxytocin receptor is a drug target for the treatment of gut pain, including some forms of IBS".

Oxytocin, often referred to as the 'love hormone', is a peptide hormone that is widely used in obstetrics and has been shown to reduce abdominal pain and increase visceral perception thresholds in the context of IBS. However, targeting druggable receptors in the gut is difficult (poor oral availability and peptide drugs are susceptible to degradation in this organ). To combat these issues, Alewood and colleagues developed more stable analogues of oxytocin and tested their efficacy in animal models of chronic abdominal pain (mice with chronic visceral hypersensitivity, CVH).
The researchers exploited the physiochemical properties of selenocysteine to improve the stability of oxytocin. The disulphide bridge in oxytocin-critical for receptor binding, but also a weak point in the structure as it renders the peptide susceptible to reduction degradation-was swapped to a selenocystathionine bond. This switch increased the half-life and stability of the peptide. "We were thrilled to see that the changes we'd made to the disulphide bridge did not affect potency or selectivity," says Alewood. Moreover, NMR structural analysis revealed distinct $3 \mathrm{D}$ structures of the oxytocin analogues in solution, indicating that the molecule is preformed ready for receptor docking.

Crucially, the researchers demonstrated that oxytocin receptors were markedly upregulated during $\mathrm{CVH}$, but not in healthy states, and that these stable oxytocin analogues had analgesic effects

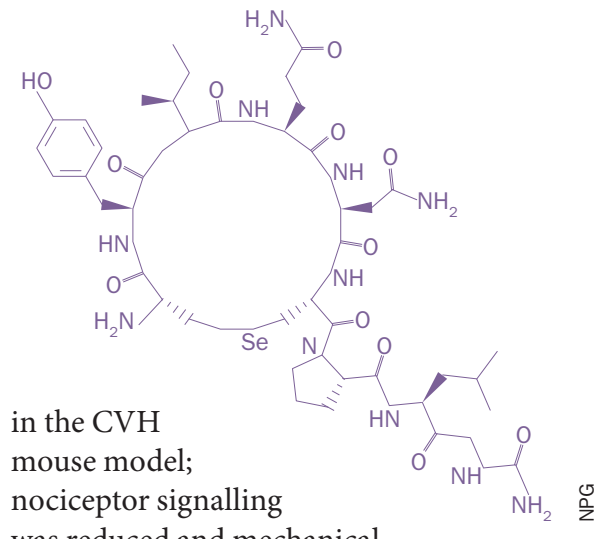

was reduced and mechanical

hypersensitivity could be reversed in vitro and in vivo, but only during states of CVH and not in healthy mice.

The new findings highlight the potential clinical use of oxytocin analogues to treat pain in the gut. The study authors are planning further research in animal models of pain in IBS and hope their work might one day progress to clinical trials.

\section{Katrina Ray}

Original article de Araujo, A. et al. Selenoether oxytocin analogues have analgesic properties in a mouse model of chronic abdominal pain. Nat. Commun. 5, 3165 (2014) 